Published in final edited form as:

Psychoneuroendocrinology. 2019 May ; 103: 125-129. doi:10.1016/j.psyneuen.2019.01.016.

\title{
Impact of mental stress, the circadian system and their interaction on human cardiovascular function
}

\author{
Frank A.J.L. Scheer ${ }^{1,{ }^{*}}$, Sarah L. Chellappa ${ }^{1}$, Kun Hu${ }^{1}$, Steve A. Shea ${ }^{1,2}$ \\ ${ }^{1}$ Medical Chronobiology Program, Division of Sleep and Circadian Disorders, Brigham and \\ Women's Hospital; and Division of Sleep Medicine, Harvard Medical School, Boston, MA 02215, \\ United States. \\ ${ }^{2}$ Oregon Institute of Occupational Health Sciences, Oregon Health \& Science University, Portland, \\ OR 97239, United States.
}

\section{SUMMARY}

The risk for adverse cardiovascular events (e.g., myocardial infarction, sudden cardiac death) peaks in the morning, possibly due to the endogenous circadian effects on cardiovascular risk factors, or the occurrence in the morning of specific triggers such as mental stress. To assess any interacting effects on cardiovascular function of mental stress and the circadian system, each of 12 healthy adults underwent a 240-h protocol with all measurements and behaviors scheduled evenly across the circadian cycle. Mental stress was repeatedly induced by performance-motivated serial addition tasks. Cardiovascular measures included hemodynamic function (heart rate, blood pressure), circulating catecholamines (epinephrine, norepinephrine), and estimates of sympathovagal balance and cardiac vagal modulation derived from heart rate variability. Mental stress increased hemodynamic function, sympathovagal balance and epinephrine, and decreased cardiac vagal modulation. Circadian variation occurred in all cardiovascular measures: sympathovagal balance peaked during the circadian morning ( $~ 9 \mathrm{AM})$, cardiac vagal modulation in the night ( $\sim \mathrm{AM})$, and heart rate and circulating catecholamines in the late morning/early afternoon ( $\sim 12 \mathrm{PM})$. Importantly, the effects of mental stress and the endogenous circadian system on cardiovascular function occurred in conjunction, such that mental stress in the circadian morning caused greatest sympathovagal balance. This summation of effects could underlie the increased morning cardiovascular vulnerability.

\section{Keywords}

Mental stress; Circadian system; Cardiovascular function; Cardiac risk factors

\footnotetext{
*Corresponding author: Frank A. J. L. Scheer, Medical Chronobiology Program, Division of Sleep and Circadian Disorders, Brigham and Women's Hospital, Boston, MA 02115, USA; Division of Sleep Medicine, Harvard Medical School, Boston, MA 02115, USA. fscheer@bwh.harvard.edu.

CONFLICT OF INTEREST

F. A. J. L. S. received speaker fees from Bayer Healthcare, Sentara Healthcare, Philips, Kellogg Company, Vanda Pharmaceuticals, and Pfizer. S.L.C., K.H., S.A.S declare no conflicts of interest.

Publisher's Disclaimer: This is a PDF file of an unedited manuscript that has been accepted for publication. As a service to our customers we are providing this early version of the manuscript. The manuscript will undergo copyediting, typesetting, and review of the resulting proof before it is published in its final citable form. Please note that during the production process errors may be discovered which could affect the content, and all legal disclaimers that apply to the journal pertain.
} 


\section{INTRODUCTION}

Major adverse cardiovascular events are the leading causes of mortality worldwide (Roth et al., 2017). Importantly, these events do not occur randomly across the day: myocardial infarction, stroke, angina, ventricular arrhythmias and sudden cardiac death display a 24-h day/night pattern with maximum risk in the morning between 6AM-12PM (Muller et al., 1985; Stergiou et al., 2002). These time-of-day variations in adverse cardiovascular events cannot be fully explained by a daily rhythm in behavioral triggers such as physical activity (Krantz et al., 1996) fasting/feeding and sleep schedules. Instead, they may also be associated with endogenous circadian changes in key aspects of cardiovascular function, including circulating catecholamines, cortisol, platelet activation, plasminogen activator inhibitor-1 (PAI-1), heart rate (HR) and autonomic nervous system activity (Scheer et al., 2010; Scheer et al., 2011; Boudreau et al., 2012; Scheer \& Shea, 2014). Importantly, there are non-additive effects of the circadian system and behavioral stressors, such that autonomic responses to exercise are greatest at a circadian phase $~ 9 A M$ (Scheer et al., 2010), and cardiovascular response to passive head-up tilt test varies across the circadian cycle (Hu et. al., 2011). Mental stress is another behavioral factor that may contribute to the morning peak in adverse cardiovascular events. Mental stress corresponds to an inner state when the demands of a situation are perceived as exceeding the personal resources of an individual at that moment (Gabbay et al., 1996; Nebel et al., 1996). Indeed, mental stress plays a role in triggering ischemia in patients with coronary artery disease (Gabbay et al., 1996).

Interestingly, the morning peak in ischemia ( $\sim \mathrm{AM})$ was accompanied by increased mental activity, while in the late evening, both reached lowest levels (Krantz et al., 1996; Nebel et al., 1996). Furthermore, acute mental stress can induce an increase in platelet activation and platelet aggregability involved in thrombosis, which is central to myocardial infarctions and ischemic strokes (Scheer et al., 2011). Collectively, these findings suggest that mental stress can increase the risk of cardiovascular events and that the magnitude of this increased risk may be modulated by the circadian timing system. Here we test the hypothesis that the effect of mental stress interacts with that of the endogenous circadian system on cardiovascular function, with maximal impact during the biological morning.

\section{METHODS}

Different aspects of this study, which was designed to test separate hypotheses, have been published previously (Scheer et al., 2010; Scheer et al., 2011; Hu et al., 2011; Scheer \& Shea, 2014).

\subsection{Participants}

Volunteers provided written informed consent, and the study was approved by the Partners Human Research Committee and performed in accordance to the Declaration of Helsinki. Twelve healthy participants (25.8 \pm 5.7 years, age range: $20-42 \mathrm{y}$; 6 women) completed the study (detailed information in Scheer et al., 2010). 


\subsection{Study protocol}

Participants spent 13 days in an individual time-free in-laboratory room at the Center for Clinical Investigation at Brigham and Women's Hospital (Boston, USA). The study protocol comprised two baseline days (16h wake/8h sleep), followed by a protocol that schedules all behaviors evenly across the circadian cycle, termed "forced desynchrony" (FD) protocol. The FD consisted of twelve 20-h 'days' (13h:20min wake, 6h:40min sleep; maintaining a 2:1 wake/sleep ratio) (Figure 1). The FD enables assessment of the separate and interacting effects of behavioral and circadian cycles, because the 20-h sleep/wake cycle is outside the range of entrainment of the human circadian system, such that the circadian system runs free at its inherent rate of $\sim 24 \mathrm{~h}$.

\subsection{Cognitive test and subjective scales}

With participants in a semi-recumbent posture, mental stress was induced by a computergenerated visual mathematical serial addition task (ADD) that involves working memory, attention and arithmetic capabilities (Burke et al., 2015). Participants were presented with a series of sequential randomly-generated pairs of 2-digit numbers and required to sum as many pairs as possible in the allotted five-minute time interval. This same ADD task was repeated twice in immediate succession within a given cognitive test session, and thus the total duration of the task was 10-min. This total duration is akin to previous work on the effects of mental stress induced by a cognitive task on cardiovascular function (Mestanik et al., 2015). The ADD task was selected due to its exquisite sensitivity to increased sleep pressure and circadian phase (Burke et al., 2015), because it can be repeatedly used over the course of a long duration study protocol (Wright et al., 2006; Burke et al., 2015), and given the mental stress effects it induces on platelet activation and platelet aggregability (Scheer et al., 2011). The same test was performed each day at $3 \mathrm{~h}: 15 \mathrm{~min}$ after scheduled awakening. Each cognitive test session was preceded by a 20-min baseline segment and followed by a 20-min recovery segment. To ensure participants were fully motivated after the first practice session, before each new session, individuals were presented with their highest number of correct responses in their previous sessions and asked to beat this performance. This standardized stressor procedure was designed to induce similar magnitudes of stress across all sessions, such that any physiological variability in cardiovascular responses to standardized stresses would be interpreted as being caused by the circadian system rather than learning or alterations in stress. After each cognitive session ( $\sim 1-2 \mathrm{~min})$, subjective stress was assessed with a visual analogue scale (where $0=$ "not stressed at all" and $100=$ "extremely stressed"), and subjective rating of performance effort was indexed by the Likert-based Performance Evaluation and Effort Rating Scales (PEERS) (Chellappa et al., 2018), using the question "The effort I had to expend for this level of performance was..." with $1=$ "Very little effort" to $4=$ "An extreme effort".

\subsection{Cardiovascular data and circadian phase assessment}

Blood pressure (BP) was measured by automatic oscillometric cuff sphygmomanometer (Dinamap; Critikon) every $5 \mathrm{~min}$ during baseline and recovery sessions and every $3 \mathrm{~min}$ during cognitive test session. To assess HR and HR variability (HRV), three-lead ECG was recorded on a Vitaport (Temec Instruments) at $256 \mathrm{~Hz}$ throughout each baseline, cognitive 
test and recovery segments. For detailed information on the cardiovascular assessments, see Scheer et al., 2010. Each cardiovascular measurement was assigned a circadian phase ( 0 $359^{\circ}$ ) based on non-orthogonal spectral analyses of continuous core body temperature recorded throughout the FD protocol, with the fitted circadian core body temperature minimum set to $0^{\circ}$ (Scheer et al., 2010).

\subsection{Statistical analyses}

Statistical analyses were performed with SAS version 9.3 (SAS Institute, Cary, NC, USA). Cardiovascular, cortisol, subjective and cognitive data were normalized based on the percentage of each subject's average for each outcome across the FD protocol, to minimize inter-individual differences in cardiovascular and behavioral function. The effects of the circadian cycle and mental stress were assessed by cosinor analyses using mixed model analyses of variance (PROC MIXED) per variable. These models included main effect "mental stress" ( 3 conditions: baseline, mental stress and recovery), a fundamental circadian component ( $24-\mathrm{h}$; effect of "circadian phase"), a harmonic component ( $12-\mathrm{h})$, and a linear component (hours into FD protocol to account for gradual changes across time). "Participant" was included as a random factor, and significance was set as a $₫ \mathbf{0} 05$.

\section{RESULTS}

We first investigated if mental stress induced acute effects on cardiovascular function (testing main effect of "mental stress"). Accordingly, mental stress significantly increased epinephrine levels by $\sim 18 \%(9 \mathrm{pg} / \mathrm{ml})(p=0.04$; see Supplementary table 1 for statistical results and post-hoc comparisons of all cardiovascular outcomes), while no significant effects were observed for norepinephrine levels. LF/HF ratio levels (sympathovagal balance) progressively increased from baseline to recovery by $\sim 25 \%$ ( $p=0.005$ ) ( 0.5 a.u.). Conversely, mental stress significantly decreased cardiac vagal modulation (HF: $\sim 40 \%$ (600ms2): $p=0.04$; RSMMD, $30 \%$ (10msec): $p=0.01 ; \mathrm{pNN} 50 \%, \sim 45 \%$ (21\%pNN50): $p=0.03$ ), with subsequent recovery. Furthermore, mental stress significantly increased hemodynamic measures [systolic BP (SBP, $\sim 2 \% ; 7 \mathrm{mmHg}$ ): $p=0.04$; diastolic BP (DBP, $\sim 5 \%, 5 \mathrm{pg} / \mathrm{ml})$ ): $p=0.04$; HR $(\sim 7 \%, 15 \mathrm{bpm}): p<0.001]$, with subsequent recovery. No significant effects of mental stress were observed for cortisol.

We then investigated whether the circadian system influences cardiovascular function during the mental stress battery (testing main effect of "circadian phase"). Sympathetic activity (epinephrine and norepinephrine) showed a significant circadian rhythm (epinephrine: $\mathrm{F}_{1,400}=27, p<0.001$; norepinephrine: $\mathrm{F}_{1,398}=4.7, p=0.03$ ), with peak levels during the late biological morning/early afternoon ( $\sim 12 \mathrm{PM}$ noon) (Figure $2 \mathrm{~A}-\mathrm{B})$. Sympathovagal balance significantly varied with circadian phase $\left(\mathrm{F}_{1,403}=4.7, p=0.02\right)$, displaying a peak during the biological morning ( $~ 9 \mathrm{AM}$ ) (Figure 2C). Cardiac vagal modulation showed a significant circadian rhythm (HF: $\mathrm{F}_{1,403}=14.4, p=0.002$; RMSSDD: $\mathrm{F}_{1,403}=24.8, p<0.001$; pNN50\%: $\mathrm{F}_{1,407}=25.1, p<0.001$ ), with a peak during the biological night ( $\left.\sim \mathrm{AM}\right)$ (Figure $2 \mathrm{D}$ and Supplementary Figure 1A-B). Hemodynamic function significantly varied with circadian phase (SBP: $\mathrm{F}_{1,412}=8.3, p=0.004$; DBP: $\mathrm{F}_{1,412}=7.1, p=0.008$; HR: $\mathrm{F}_{1,412}=81.5, p<0.001$ ), with maximum levels at the circadian phase corresponding to the biological evening ( $\sim 9 \mathrm{PM})$

Psychoneuroendocrinology. Author manuscript; available in PMC 2020 May 01. 
for $\mathrm{BP}$ and to the late biological morning/early afternoon ( $\sim 12 \mathrm{PM}$ noon) for HR (Figure 2E$\mathrm{G})$. Furthermore, cortisol significantly varied with circadian phase $\left(\mathrm{F}_{1,401}=409, \mathrm{p}<0.001\right)$, peaking during the biological morning ( $\sim 9 \mathrm{AM})$.

No significant interactions between the effect of the mental stress battery and circadian phase were observed for the cardiovascular outcomes. Furthermore, we repeated all cosinor analyses on absolute (raw) data and observed similar results. Lastly, we assessed whether subjective stress effects (subjective rating of performance effort) and cognitive effects (objective task performance) predicted any of our cardiovascular outcomes (Supplementary Figure 2A-C). Subjective ratings of stress and performance effort showed no significant circadian phase effects, while a statistical trend was observed for the circadian influence on cognitive performance $\left(\mathrm{F}_{1,127}=3.8, p=0.052\right)$. Furthermore, none of these stress and cognitive markers significantly predicted circadian fluctuations in cardiovascular function (covariance analyses on normalized and raw data).

\section{DISCUSSION}

Our data show that mental stress and the endogenous circadian system impact on key aspects of human cardiovascular function. Previous data on cardiovascular reactivity in response to different acute mental stressors showed increased HR, BP and cardio-sympathetic response especially for the demanding (high cognitive load) Stroop task (Mestanik et al., 2015). Furthermore, greater reactivity to and reduced recovery from mental stress may be associated with poor cardiovascular status, including hypertension and increased carotid thickness (for a review, see Chida \& Steptoe, 2010). Potential mechanisms for cardiovascular reactivity to mental stress include a complex interaction of neural mechanisms regulating cognitive and affective functions, forming a system that coordinates the psychophysiological demands of these neural units with the autonomic cardiac response to environmental challenges (Mestanik et al., 2015). A caveat to these studies is that cardiovascular reactivity to mental stress was limited to a single assessment during the day or only in the morning and afternoon (Nebel et al., 1996). It is well-known that human cardiovascular function fluctuates across the day, plausibly due to changes in response to, e.g., sleep/wake, physical activity, postural changes, and/or due to endogenous circadian changes in vascular tone, catecholamines, PAI-1, HR and HRV (Scheer et al., 2010; Boudreau et al., 2012; Scheer \& Shea, 2014). Thus, one may hypothesize that cardiovascular responses induced by mental stress fluctuate over time and that these effects may interact with those of the endogenous circadian system. Adverse cardiovascular events exhibit maximum risk in the morning hours (Muller et al., 1985; Stergiou et al., 2002). Here we show that, of all outcomes of cardiovascular function, only sympathovagal balance clearly peaks during the circadian morning, while HR, epinephrine and norepinephrine display rapid rises across the vulnerable morning window, and peak slightly afterwards. However, caution is warranted when using LF/HF as a reflection of sympathovagal balance (Billman et al, 2015) due to its complex physiological origins (Berntson et al, 1997), i.e., LF is influenced not only by the sympathetic nervous system but also by the parasympathetic system.

The circadian rise in catecholamines may contribute to increased morning risk, such that, after low levels during the circadian night, there may be upregulation of adrenergic receptors 
(Witte et al., 1995). Given the effects of epinephrine on blood clotting, myocardial oxygen demand and vasoconstriction, it seems possible that in vulnerable individuals, the large circadian epinephrine rhythm, together with the circadian rhythm in sympathovagal activity, could contribute to the morning peak in adverse cardiovascular events. Importantly, our data indicate that both mental stress and circadian effects have effects on key hemodynamic, sympathovagal, cardiac vagal modulation and sympathetic activity markers. We previously showed that mental stress can induce increases in markers of platelet function and that these effects also do not show an interaction with the circadian control (Scheer et al., 2011).

Therefore, if an individual experiences mental stress during the "vulnerable" morning hours associated to adverse cardiovascular risk, they may be exposing themselves to an added risk. The effects of standardized laboratory-induced mental stress and the circadian system were smaller that the effects of physical stressors, such as exercise (Scheer et al., 2010) and headup tilt (Hu et al., 2011). However, they do occur and could contribute to a vulnerable state. Potential limitations to our study include the absence of a test of a dose-dependent mental stress effect, as more challenging cognitive tasks may elicit stronger cardiovascular reactivity (Mestanik et al., 2015). While the addition task may be a mild mental stressor, it was selected to allow for repeated exposure every $20 \mathrm{~h}$ and to not to influence subsequent tests (Scheer et al., 2011). Future studies are required to test whether exposure to more demanding tasks may uncover an interaction with circadian phase on cardiovascular reactivity. Ultimately, our findings suggest that the combined effects on cardiovascular reactivity of mental stress and the endogenous circadian system should be considered when assessing increased individual risk during the vulnerable morning hours.

\section{Supplementary Material}

Refer to Web version on PubMed Central for supplementary material.

\section{ACKNOWLEDGEMENTS}

We thank research volunteers and Brigham and Women's Hospital's Center for Clinical Investigation nursing and technical staff, and Dr. Wei Wang for statistical advice. This study did not involve off-label or investigational drug use. This study is not a clinical trial.

FUNDING

This work was supported by the National Institutes of Health [grant numbers R01HL76409 and NCRR-GCRCM01-RR02635]. SC was supported in part by R01HL118601. SAS was supported in part by K24HL076446, R01HL125893 and the Oregon Institute of Occupational Health Sciences. FAJLS was supported in part by R01HL118601, R01DK099512, R01DK102696, R01DK105072, and R01HL140574.

\section{REFERENCES}

Berntson GG et al. 1997 Heart rate variability: Origins, methods, and interpretive caveats. Psychophysiology. 34, 623-648. [PubMed: 9401419]

Billman GE et al. 2015 An introduction to heart rate variability: methodological considerations and clinical applications. Front Physiol 25(6), 1-3.

Boudreau P et al. 2012 A circadian rhythm in heart rate variability contributes to the increased cardiac sympathovagal response to awakening in the morning. Chronobiol Int 29, 757-768. [PubMed: 22734576]

Chellappa SL et al. 2018 Daily circadian misalignment impairs human cognitive performance taskdependently. Sci Rep 8(1), e3041. 
Chida Y \& Steptoe A 2010 Greater cardiovascular responses to laboratory mental stress are associated with poor subsequent cardiovascular risk status: a meta-analysis of prospective evidence. Hypertension. 55(4), 1026-1032. [PubMed: 20194301]

Gabbay FH et al. 1996 Triggers of myocardial ischemia during daily life in patients with coronary artery disease: physical and mental activities, anger and smoking. J Am Coll Cardiol 27(3), 585592. [PubMed: 8606268]

Krantz DS et al. 1996 Circadian variation of ambulatory myocardial ischemia. Triggering by daily activities and evidence for an endogenous circadian component. Circulation. 93(7), 1364-1371. [PubMed: 8641025]

$\mathrm{Hu} \mathrm{K}$ et al. 2011 Endogenous circadian rhythm in vasovagal response to head-up tilt. Circulation. 123(9), 961-970. [PubMed: 21339480]

Mestanik M 2015 Cardiovascular sympathetic arousal in response to different mental stressors. Physiol Res 64(5), 585-594.

Muller JE et al., 1985 Circadian variation in the frequency of onset of acute myocardial infarction. N Engl J Med 313, 1315-1322. [PubMed: 2865677]

Nebel LE et al. 1996 The circadian variation of cardiovascular stress levels and reactivity: relationship to individual differences in morningness/eveningness. Psychophysiology. 33(3), 273-281. [PubMed: 8936396]

Roth GA et al. 2017 Global, Regional, and National Burden of Cardiovascular Diseases for 10 Causes, 1990 to 2015. J Am Col Cardiol 70(1), 1-25.

Scheer FA \& Shea SA 2014 Human circadian system causes a morning peak in prothrombotic plasminogen activator inhibitor-1 (PAI-1) independent of the sleep/wake cycle. Blood. 123(4), 590-593. [PubMed: 24200683]

Scheer FA et al. 2011 The human endogenous circadian system causes greatest platelet activation during the biological morning independent of behaviors. PLoS One. 6(9), e24549. [PubMed: 21931750]

Scheer FA et al. 2010 Impact of the human circadian system, exercise, and their interaction on cardiovascular function. Proc Natl Acad Sci U S A. 107(47), 20541-20546. [PubMed: 21059915]

Stergiou GS el al., 2002 Parallel morning and evening surge in stroke onset, blood pressure, and physical activity. Stroke. 33, 1480-1486. [PubMed: 12052978]

Witte K et al. 1995 Mechanisms of the circadian regulation of beta-adrenoceptor density and adenylyl cyclase activity in cardiac tissue from normotensive and spontaneously hypertensive rats. J Mol Cell Cardiol 27, 1195-1202. [PubMed: 7473777] 


\section{Highlights}

- Mental stress impacts on cardiovascular function.

- Cardiovascular function shows robust circadian variations

- Mental stress and circadian effects on cardiovascular function are additive 


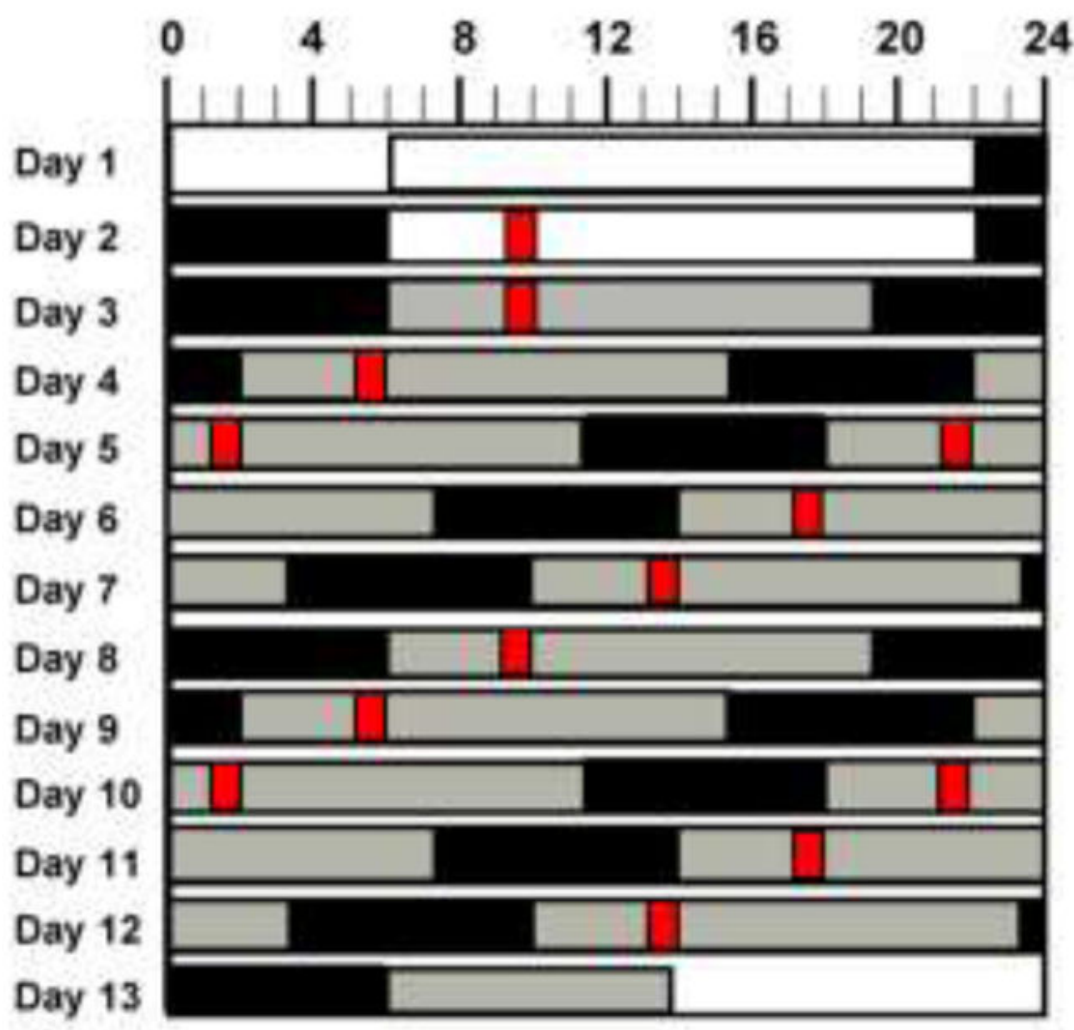

Figure 1. Study design.

Graphical representation of the forced desynchrony protocol. Solid black areas indicate scheduled sleep in darkness (0 lux); light gray, wakefulness in dim light ( $\sim 1.8$ lux); white bars, wakefulness on baseline days in normal room light ( 90 lux); and narrow red bars, scheduled cognitive test (including baseline, visual addition task and recovery periods). Subject's habitual bedtime is 10PM in this example. 


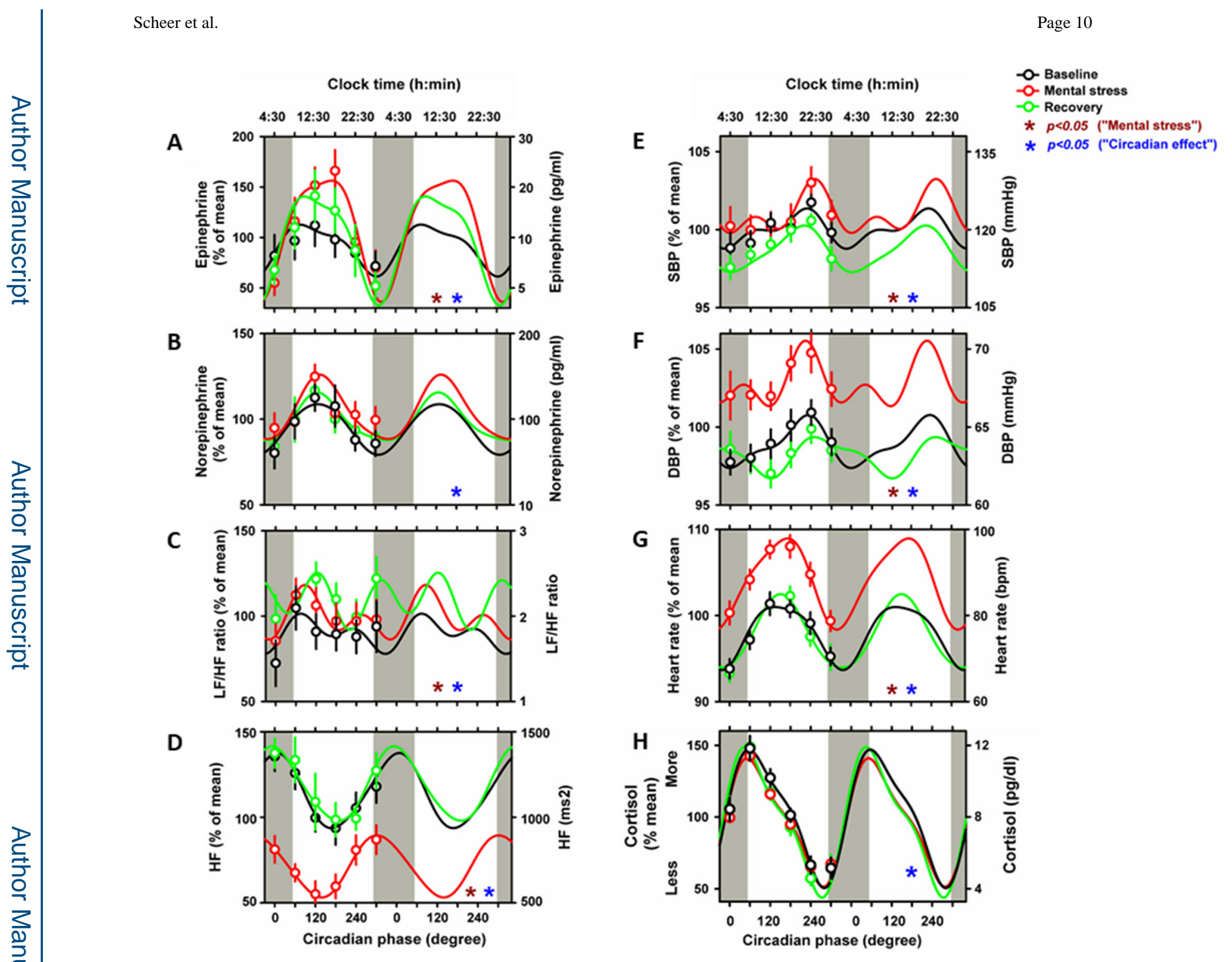

Figure 2. Mental stress and circadian effects on human cardiovascular function.

Cosinor models of circadian rhythms of Epinephrine $(\mathbf{A})$, norepinephrine $(\mathbf{B}), \mathrm{LF} / \mathrm{HF}$ ratio (C), HF power (D), SBP (E), DBP (F), heart rate $(\mathbf{G})$ and cortisol (H) during baseline (black lines), mental stress (red lines) and recovery (green lines). Cosinor analyses were performed on the $360^{\circ}$ data sets, whereas data are double plotted (two identical circadian cycles) to aid visualization of rhythmicity. To show that these models adequately fit the actual data, we also plot average data grouped into 60 circadian degree bins with SEM error bars (averaged data are not double plotted). Bottom $x$-axes, circadian phase with $0^{\circ}$ indicating circadian core body temperature minimum (average: $\sim 4 \mathrm{AM}$ ); top $\mathrm{x}$-axes, corresponding average clock time; left y-axes, percentage of each participant's mean across the days under forced desynchrony; right y-axes, absolute values. Maroon stars: "mental stress effect"; Blue stars: "circadian effect"; $p<0.05$. 